\title{
When is dental neglect a safeguarding concern?
}

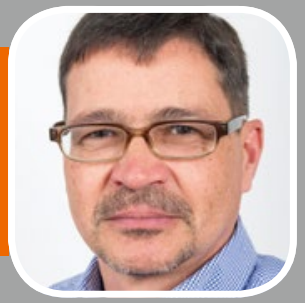

Mark Foster of the Child Protection
Company puts dental neglect
into context and examines our
responsibilities as dental professionals.

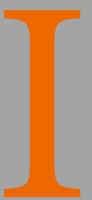

$\mathrm{n}$ an ideal world, safeguarding training wouldn't need to exist because abuse and neglect simply wouldn't affect us. However, the unfortunate truth is that hundreds of thousands of individuals fall victim to abuse and neglect all across the United Kingdom every year - and of course, these are only the reported cases. It's likely that there are many more victims of abuse and neglect who remain unaccounted for.

It's because of this that you have a duty as a dental professional to ensure safeguarding training is kept up to date at your practice. Safeguarding is the act of protecting people from abuse and neglect by putting measures in place to ensure their safe and effective care. Closely linked and included in your safeguarding training is child protection, which is the process we go through after a safeguarding incident has been identified, involving measures to protect individuals from any further harm.

You're required to know exactly how to spot a potential safeguarding concern and to understand where to report what you've seen, no matter if you witness it happening at work or out of hours. It doesn't matter your role within the dental practice either. Safeguarding is everyone's responsibility, and everyone in a customer facing role within the practice will need to complete at least a Level 1 dental safeguarding course to fulfil the Care Quality Commission's inspection guidelines. For those working in a clinical capacity a Level 2 dental safeguarding course is also required.
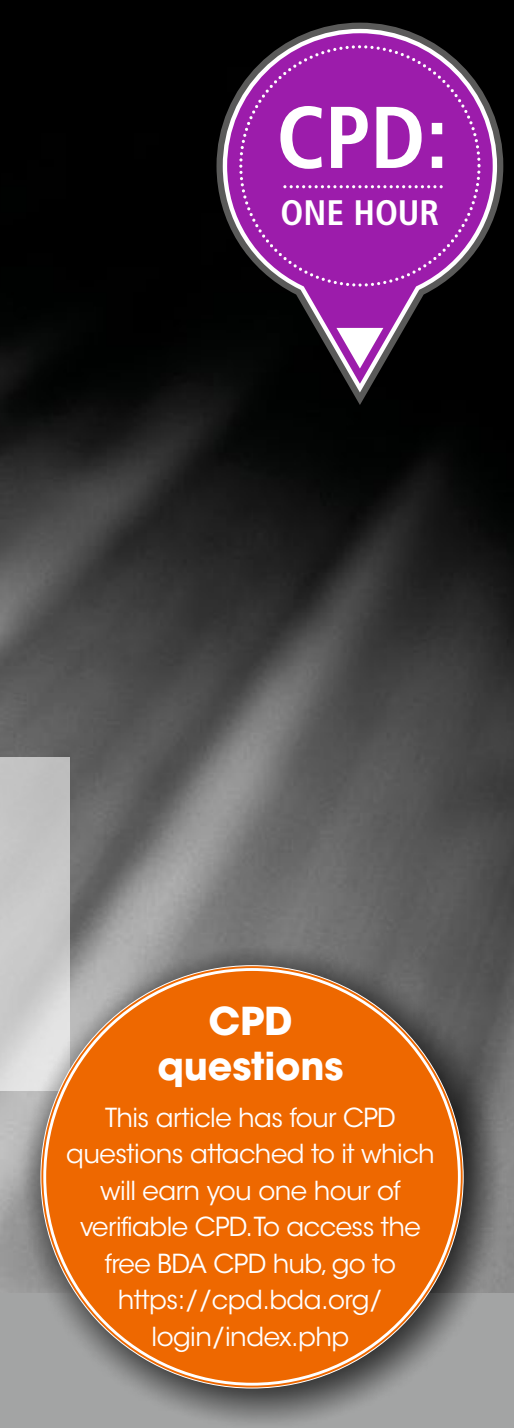

A focus on dental neglect

You probably know all of this already, but there are some elements of safeguarding that are unique to the dental sector, which you should be refreshing your knowledge of periodically to ensure that you're up to date with the latest practice. Dental neglect is one of these topics.

The British Society of Paediatric Dentistry (BSPD) defines dental neglect as the following: 'The persistent failure to meet a child's basic oral health needs, likely to result in the serious impairment of a child's oral or general health or development'.

Indeed, dental neglect is a very serious issue, and in some cases, it can be one of the first steps towards identifying wider neglect or abuse. While the definition above focuses on children, it's important to remember that this information applies to individuals of all ages. That includes vulnerable adults such as those with learning disabilities, the elderly, and anyone else who is dependent on another person to help them with daily life to any extent. 
Because of your position in the dental practice, you could be one of the only people to notice when something isn't quite right if a patient's case of dental neglect turns out to be an identifying feature of underlying abuse. Understandably then, you need to know how to respond to cases of dental neglect responsibly and sensitively, with a view to a patient's overall wellbeing and safety and not only with concerns for their oral health.

A dental-specific safeguarding training course such as the Child Protection Company's online Introduction to Adult/ Child Protection (recommended by the British Dental Association and available to buy as an individual or as part of a team at www.childprotectioncompany.com) will teach you more about dental neglect and how to notice when something isn't quite right.

\section{Putting dental neglect into context}

Part of your duty when it comes to safeguarding is knowing when to trust your own judgement and to follow your gut instinct to report a concern. Current guidance reminds dental professionals that there is never such a thing as being 'wrong' when it comes to reporting safeguarding concerns. In the event that abuse is uncovered, you have helped to take a victim out of harm's way; if it turns out after investigation that there isn't any cause for concern, then you have the peace of mind that the individual in question is safe.

However, it's never easy to know when to act. It's a worrying truth, but many perpetrators of abuse are skilled at hiding their actions and can use tactics such as manipulation, threats, blackmail, or any other emotional or physical force to keep their actions a secret between themselves and their victims. It's true that many perpetrators of abuse can even come across as highly unsuspecting, with charming personalities and an eager to please attitude, happy to do anything at all to build a rapport with you.

Of course, all of this and more can make it extra difficult for you to be sure if there are any underlying safeguarding concerns related to a patient's dental neglect. But you must be trained to see past the façade and know that these charming personas are often little more than a mask to disguise an abuser's true intentions. Any case of dental neglect should ring alarm bells and should not be ignored.

If you're having doubts about whether to act upon a safeguarding concern related to dental neglect, put the neglect into context and ask yourself questions such as:

- Do you know much about the patient's family/home life?
- Have you seen any other concerning behaviour from this individual or any of their relatives/carers/parents?

- Could a lack of access to treatment be the reason for the dental neglect (ie if the individual has recently moved to the UK from a country where dental care is not easily accessible or affordable)?

- How frequently are you noticing issues with the patient's oral hygiene?

- Does the patient or their carer appear to have acted on any of your previous advice related to oral health if the neglect has been noticed before?

- Has the patient missed any appointments with you?

- What is the impact of the dental neglect on the patient's health?

- Do you know any other information about the patient that might explain their reasons for neglecting oral health?

If you still have any concerns for the patient's wellbeing after answering these questions, no matter how small or seemingly insignificant, you should report the information you have by following your practice's standard safeguarding procedure.

Always keep in mind that this information might be just one more nugget in an ongoing case, and it's very unlikely that anyone else in the patient's life will have an opportunity to assess or notice their case of dental neglect. You are uniquely positioned to share this insight as a dental professional, so if you feel a person's wellbeing is at risk, you should not hesitate to escalate your concerns.

\section{Adapting how we speak about dental neglect}

In the event of any safeguarding concern, it's important to maintain a person-centred focus. This means taking into consideration the victim's thoughts and feelings before any other individual's and allowing the victim to have a voice in the investigation.

We can encourage a person-centred focus by adapting the language we use when it comes to dental neglect, including that surrounding missed appointments. After all, if a person is consistently missing their dental appointments, this is a cause for concern in itself even if they display no other signs of neglect to their oral health.

Previously, we might have said that a patient 'Did Not Attend' their dental appointment, but the British Dental Association is calling on dental professionals to change their language and instead say that a patient 'Was Not Brought' to their appointment. This applies primarily to patients who are children, the elderly, disabled, or otherwise dependent on somebody else, such as a parent or carer, to bring them to their appointment.

When we say that a patient 'Did Not Attend' their dental appointment, this adds an element of personal choice into the mix that might not necessarily reflect what is really happening. Realistically, it's unlikely that a child would have the final say in whether or not they attend their dental appointments, for example, simply because they would not be able to bring themselves along to the practice without being accompanied by an adult. So, when we use the phrase 'Was Not Brought', we're including the possibility of other individuals in the patient's life impacting their decision to miss an appointment or neglecting to bring them to the dentist.

Simple changes like this can help us to keep the focus on the patient who might potentially be at risk, rather than assuming a missed appointment is automatically their fault. For any number of reasons, a missed appointment could be a genuine innocent mistake, but if a patient is consistently not turning up, or if they have no valid reason and give an unbelievable or otherwise suspicious excuse when asked why, you shouldn't rule out the possibility that this could be a safeguarding concern.

\section{Learn more about dental safeguarding}

If you'd like to learn more about dental safeguarding, or to arrange verifiable, dental-specific safeguarding training with the Child Protection Company, please visit our website at www.childprotectioncompany.com, where you can browse our British Dental Association-recommended online courses.

Alternatively, why not get in touch with our friendly customer support team for a tailor-made quote by giving us a call on 01327 552030,or email help@ childprotectioncompany.com. Our offices are open from 9 am to $5.30 \mathrm{pm}$, Monday to Friday, and we're always happy to help.

\section{CPD questions}

This article has four CPD questions attached to it which will earn you one hour of verifiable CPD. To access the free BDA CPD hub, go to https://cpd.bda.org/login/index.php.

https://doi.org/10.1038/s41407-020-0256-6 